\section{(C) OPEN ACCESS}

\title{
DNA methylation mapping identifies gene regulatory effects in patients with systemic lupus erythematosus
}

Juliana Imgenberg-Kreuz, 1,2 Jonas Carlsson Almlöf, ${ }^{1}$ Dag Leonard, ${ }^{2}$ Andrei Alexsson, ${ }^{2}$ Gunnel Nordmark, ${ }^{2}$ Maija-Leena Eloranta, ${ }^{2}$ Solbritt Rantapää-Dahlqvist, ${ }^{3}$ Anders A Bengtsson, ${ }^{4}$ Andreas Jönsen, ${ }^{4}$ Leonid Padyukov, ${ }^{5}$ Iva Gunnarsson, ${ }^{5}$ Elisabet Svenungsson, ${ }^{5}$ Christopher Sjöwall, ${ }^{6}$ Lars Rönnblom, ${ }^{2}$ Ann-Christine Syvänen, ${ }^{1}$ Johanna K Sandling ${ }^{1,2}$

\begin{abstract}
Handling editor Josef S Smolen

- Additional material is published online only. To view please visit the journal online (http://dx.doi.org/10.1136/ annrheumdis-2017-212379).
\end{abstract}

${ }^{1}$ Department of Medical Sciences, Molecular Medicine and Science for Life Laboratory, Uppsala University, Uppsala, Sweden

${ }^{2}$ Section of Rheumatology, Department of Medical Sciences, Uppsala University, Upssala, Sweden

${ }^{3}$ Department of Public Health and Clinical Medicine/

Rheumatology, Umeå University, Umeå, Sweden

${ }^{4}$ Department of Clinical

Sciences, Section of

Rheumatology, Lund University, Skane University Hospital, Lund, Sweden

${ }^{5}$ Rheumatology Unit, Department of Medicine Solna, Karolinska Institutet, Karolinska University Hospital, Stockholm, Sweden

${ }^{6}$ Department of Clinical and Experimental Medicine, Rheumatology/Division of Neuro and Inflammation Sciences, Linköping University, Linköping, Sweden

Correspondence to Dr Johanna K Sandling, Department of Medical Sciences, Uppsala University, Rudbeck Laboratory, Uppsala 75185 , Sweden;

johanna.sandling@medsci.uu.se

Received 12 September 2017 Revised 11 January 2018 Accepted 15 January 2018 Published Online First 1 February 2018

\section{Check for updates}

To cite: Imgenberg-Kreuz J, Carlsson Almlöf J, Leonard D, et al. Ann Rheum Dis 2018;77:736-743

\section{ABSTRACT}

Objectives Systemic lupus erythematosus (SLE) is a chronic autoimmune condition with heterogeneous presentation and complex aetiology where DNA methylation changes are emerging as a contributing factor. In order to discover novel epigenetic associations and investigate their relationship to genetic risk for SLE, we analysed DNA methylation profiles in a large collection of patients with SLE and healthy individuals. Methods DNA extracted from blood from 548 patients with SLE and 587 healthy controls were analysed on the Illumina HumanMethylation $450 \mathrm{k}$ BeadChip, which targets $485000 \mathrm{CpG}$ sites across the genome. Single nucleotide polymorphism (SNP) genotype data for 196 524 SNPs on the Illumina ImmunoChip from the same individuals were utilised for methylation quantitative trait loci (cis-meQTLs) analyses.

Results We identified and replicated differentially methylated CpGs (DMCs) in SLE at 7245 CpG sites in the genome. The largest methylation differences were observed at type I interferon-regulated genes which exhibited decreased methylation in SLE. We mapped cismeQTLs and identified genetic regulation of methylation levels at 466 of the DMCs in SLE. The meQTLs for DMCs in SLE were enriched for genetic association to SLE, and included seven SLE genome-wide association study (GWAS) loci: PTPRC (CD45), MHC-class III, UHRF1BP1, IRF5, IRF7, IKZF3 and UBE2L3. In addition, we observed association between genotype and variance of methylation at 20 DMCs in SLE, including at the HLA$D Q B 2$ locus.

Conclusions Our results suggest that several of the genetic risk variants for SLE may exert their influence on the phenotype through alteration of DNA methylation levels at regulatory regions of target genes.

\section{INTRODUCTION}

Systemic lupus erythematosus (SLE, MIM 152700) is an autoimmune disease characterised by defective clearance of apoptotic cells, production of a large number of different autoantibodies and activation of the type I interferon (IFN) system. ${ }^{12}$ So far, more than 80 genetic loci that contribute to SLE susceptibility have been identified. ${ }^{34}$ Both candidate gene and genome-wide association studies (GWAS) have provided insights into the affected signalling pathways, but do not fully explain the genetic susceptibility to SLE. ${ }^{5} 6$
Epigenetic regulation is emerging as an important contributing factor in SLE. Promoter demethylation in lymphocytes leading to overexpression has been reported for several SLE candidate genes, as has global DNA hypomethylation in lymphocytes in patients with SLE. ${ }^{7-9}$ In addition, demethylating agents are known to cause drug-induced lupus. ${ }^{10}$ Using the Illumina HM450k BeadChip to analyse fractionated blood cells from patients with SLE and healthy controls, Absher et al and Coit et al report hypomethylation at type I IFN system genes across all tested blood cell types. ${ }^{11} 12$ These studies indicate a role for DNA methylation in regulating the type I IFN system in SLE. Associations between DNA methylation and different manifestations of SLE have also been reported, and they include autoantibody production, nephritis and skin rash. ${ }^{13-16}$ However, these findings are yet to be independently replicated.

In order to discover novel epigenetic associations in SLE, we generated genome-wide methylation profiles from a large collection of Swedish patients with SLE and healthy controls. To date, there have been no large-scale studies that investigate the role of genetics in regulating DNA methylation levels and variance of DNA methylation in SLE and the effect of these measures on SLE susceptibility. Therefore, we intersected our genome-wide DNA methylation data with genetic data on the same cohorts to identify gene regulatory effects on DNA methylation in SLE.

\section{METHODS}

For full details of methods see online Supplementary file 1.

\section{Subjects and samples}

In the discovery phase, patients with SLE visiting university hospitals in Uppsala and Linköping, ${ }^{17}$ Sweden $(n=400)$, and control individuals from the Uppsala BioResource $(n=400)$ of healthy blood donors were included. As replication, patients with SLE $(n=201)$ and controls $(n=187)$ from the Karolinska University hospital in Stockholm, Sweden, were included. All subjects provided informed consent to participate in the study. Five hundred and forty-eight patients fulfilling at least four of the eleven 1982 American College for Rheumatology (ACR) criteria for SLE $^{18}$ were included in the subsequent analyses. 


\section{DNA methylation analysis}

DNA methylation levels of 485577 CpG (C-phosphate-G) sites were determined using the HM450k BeadChip (Illumina, San Diego, California, USA). ${ }^{19}$ Signal intensities were parsed into the Minfi R package for quality control (QC) and Subset-quantile Within Array Normalisation. ${ }^{20-22}$ The post-QC dataset comprised $385851 \mathrm{CpG}$ sites, 347 patients with SLE and 400 controls for the discovery phase and 201 patients and 188 controls for the replication phase. The aggregate of methylation beta values for all $\mathrm{CpG}$ sites followed identical bimodal distributions in both cases and controls (see figure S1 in the online Supplementary file 2).

\section{Genotyping}

Quality controlled genotype data for 133838 SNPs generated on the Infinium ImmunoChip (Illumina) ${ }^{23}$ were available for 527 patients with SLE and 567 of the healthy control individuals with HM450k data. The SLE case-control genetic association analysis included a larger set of 1135 Swedish patients with SLE and 2931 Swedish control individuals from the university hospital rheumatology clinics at Uppsala, Stockholm Karolinska Solna, Linköping, Lund, and the four northernmost counties of Sweden.

\section{Epigenome-wide association analysis}

Relative blood cell composition of the samples was determined using the method by Houseman et $a l^{24}$ (see figure S2 in the online Supplementary file 3). To determine differential methylation between patients with SLE and controls, a linear regression model was fitted. Differentially methylated $\mathrm{CpG}$ sites (DMCs) were called in the discovery phase if they had a $\mathrm{P}<1.3 \times 10^{-7}$ for association based on Bonferroni correction and an absolute average difference in methylation beta values between cases and controls of $>0.05$. Significance in the replication phase was determined as $\mathrm{P}<0.05$ divided by the number of tested $\mathrm{CpG}$ sites and same direction of effect. Similarly, the role of methylation in different disease manifestations was investigated in a case-case analysis as was the association between different medications and methylation.

\section{Methylation quantitative trait loci (meQTL) analysis}

Methylation levels were tested in PLINK for genotype association separately in patients and controls assuming an additive model. ${ }^{25}$ A Bonferroni corrected $\alpha<0.05$ was considered significant. Methylation variance was calculated as the difference between a subject's methylation value and the genotype-specific mean.

\section{RESULTS}

\section{Genome-wide DNA methylation patterns in SLE}

We used the Illumina HumanMethylation $450 \mathrm{k}$ BeadChip ${ }^{19}$ and analysed methylation levels at $385851 \mathrm{CpG}$ sites across the human genome in an epigenome-wide association study (EWAS) for SLE in genomic DNA from whole blood. The study included a total of 548 patients with SLE and 588 age-matched and gendermatched controls, and we employed a discovery and replication phase study design (see table S1 in the online Supplementary file 4). In the discovery phase, we identified 7625 DMCs using logistic regression in patients with SLE compared with controls at a Bonferroni corrected $\mathrm{P}$ value $<1.3 \times 10^{-7}$ and average methylation difference $|\Delta \beta|>0.05$ (figure 1 ; see table $S 2$ in the online Supplementary file 5). The vast majority of the DMCs identified in the discovery cohort exhibited decreased DNA methylation levels in patients with SLE compared with controls (75\%; 5717 of $7625 \mathrm{CpG}$ sites). As many as 7245 DMCs (95\%) replicated in the second cohort (Bonferroni corrected P value $<6.6 \times 10^{-6}$ ) (see table S2 in the online Supplementary file 5). A noteworthy result from the genome-wide DNA methylation analysis is that we observed large differential methylation of $|\Delta \beta|>0.1$ almost exclusively at IFN-regulated genes (table 1). This epigenetic IFN pattern was observed both in patients with active and inactive disease, although the effect was more prominent in active SLE (see table S3 in the online Supplementary file 6). The CpG site with the largest increased methylation in SLE was cg08450017 in CXCR6, which is involved in $\mathrm{C}-\mathrm{X}-\mathrm{C}$ chemokine signalling and whose ligand CXCL16 is elevated in SLE serum and has been suggested as a biomarker in SLE (figure 2; see table S2 in the online Supplementary file 5). ${ }^{26} 27$

A total of 4034 of the replicated DMCs in SLE that we identify in blood cells in patients with SLE are novel and are annotated to 1638 unique genes that have to our knowledge not previously been linked with DNA methylation in SLE. ${ }^{11} 122829$ Among the most significant novel DMCs in SLE we note cg03889044 in PDCD1, which is a confirmed SLE susceptibility locus. ${ }^{30} 31$ PDCD1 encodes the Programmed Cell Death 1 (PD-1) protein that functions in preventing autoimmunity by inhibiting activation of self-reactive lymphocytes. ${ }^{32}$ Another example of a previously unreported DMC in SLE is cg24414363 in centromere protein $M$ (CENPM). CENPM is involved in regulating cell division processes and is preferentially expressed in activated lymphocytes. ${ }^{33}$ We further identified highly significant novel DMCs in SLE at the genes adenylate kinase $2(A K 2)$ playing a role in apoptotic processes and activating signal cointegrator 1 complex subunit 2 (ASCC2) involved in transcriptional regulation.

To further characterise our most significant DMCs in SLE, we performed gene ontology enrichment analysis for the most significant and replicated DMCs annotated to genes. We found that genes which had DMCs in SLE were highly enriched in the molecular functions enzyme binding, regulatory region DNA binding and transcription factor activity, as well as in biological processes related to leucocyte activation (table 2, see table S4 in the online Supplementary file 7). Additionally, we found that the replicated DMCs in SLE were depleted in CpG islands, but were enriched in regions with a histone mark for active enhancers (H3K4me1) in B and T cells (see figure S3 in the online Supplementary file 8 ).

To avoid confounding due to gender differences in DNA methylation patterns, $\mathrm{CpG}$ sites located on the sex chromosomes were analysed separately in females and males. In females, we replicated 27 X-chromosomal DMCs in SLE (see table S5 in the online Supplementary file 9). These DMCs were annotated to several genes implicated in immune cell function, such as TLR8 involved in pathogen recognition and VSIG4, a negative regulator of T-cell proliferation. In males, there were three replicated $\mathrm{X}$-chromosomal DMCs in SLE; these were annotated to the SH2D1A and SEPT6 genes and an intergenic region, respectively (see table S6 in the online Supplementary file 10). SH2D1A plays a role in stimulation of $\mathrm{T}$ and $\mathrm{B}$ cells and septin 6 is required for cytokinesis.

\section{Methylation changes associated with SLE disease manifestations}

As SLE is a clinically heterogeneous disease, we compared the DNA methylation levels between patients that display a specific disease manifestation defined in the ACR 1982 classification 


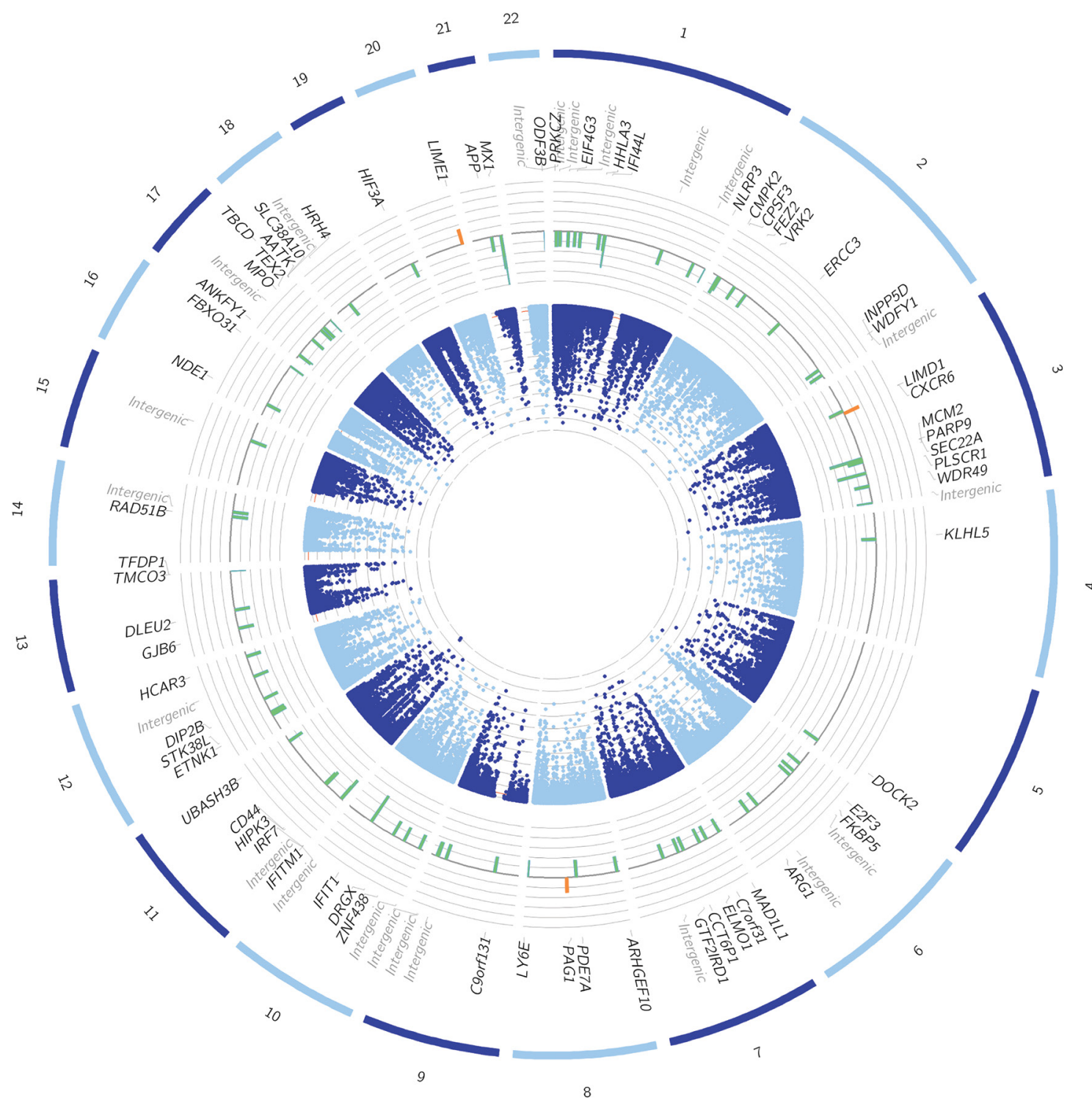

Figure 1 Results from the case-control epigenome-wide association study (EWAS) in systemic lupus erythematosus (SLE) in the discovery cohort. Inner circle: circular Manhattan plot of the results of the SLE case-control association analysis. P values are presented on the -log10 scale where the innermost scale line represents $10^{-214}$. Middle circle: average methylation difference $(\Delta \beta)$ between patients with SLE and controls for the top 100 differentially methylated $\mathrm{CpG}$ sites in the EWAS (scale -0.4 to 0.4 ). Green bars indicate decreased methylation and orange bars represent increased methylation levels in patients compared with controls. The outer circle represents chromosomes 1-22.

criteria for $\operatorname{SLE}^{18}$ against the remaining patients lacking this disease manifestation (see table S1 in the online Supplementary file 4). We were only able to identify a total of 49 DMCs associated with ACR criteria for SLE in the discovery cohort $\left(\mathrm{P}\right.$ value $\left.<1.3 \times 10^{-7},|\Delta \beta|>0.05\right)$ (see table $\mathrm{S} 7$ in the online Supplementary file 11). None of these 49 DMCs reached the corrected significance threshold in the replication cohort.

\section{Methylation changes associated with SLE treatment}

As a majority of the patients with SLE received treatment to control their disease at the time of blood sampling, we investigated whether methylation levels were associated with the most commonly prescribed medications. By comparing patients that received a specific medication at blood sampling to those who did not, we identified and replicated 5321 DMCs for medication in SLE when correcting for disease activity (see table S8 in the online Supplementary file 12). The overwhelming majority of the DMCs for medication were observed for glucocorticoid treatment $(n=5196)$, which typically was associated with decreased methylation.

Due to the large number of $\mathrm{CpG}$ sites associated with glucocorticoid treatment, we repeated the SLE case-control methylation analyses in the subsets of patients who were not receiving glucocorticoid treatment at the time of blood sampling (discovery $\mathrm{n}=132$ and replication $\mathrm{n}=89$ ). Of the 7245 replicated DMCs in SLE, 3295 were also significant in this analysis applying Bonferroni correction for multiple testing, and 6411 reached nominal significance $(\mathrm{P}<0.05)$ in both cohorts with the same direction of the effect (see table S9 in the online Supplementary file 13 and figure S4 in the online Supplementary file 14).

\section{Genetic regulation of DNA methylation in SLE}

To search for cis-acting genetic variants that regulate DNA methylation in SLE, we analysed DNA methylation levels against the genotypes of single nucleotide polymorphisms (SNPs) in risk loci for autoimmune diseases in a cis-meQTL analysis (see figure S5 
Table 1 Top differentially methylated CpG sites (DMCs) in the systemic lupus erythematosus case-control association analysis

\begin{tabular}{|c|c|c|c|c|c|c|c|c|}
\hline \multirow[b]{2}{*}{ Chromosome } & \multirow[b]{2}{*}{ Position } & \multirow[b]{2}{*}{ CpG site } & \multirow[b]{2}{*}{ Gene } & \multirow{2}{*}{$\begin{array}{l}\text { Interferon } \\
\text { induced* }\end{array}$} & \multicolumn{2}{|l|}{ Discovery } & \multicolumn{2}{|c|}{ Replication } \\
\hline & & & & & $P$ valuet & Methylation $\Delta \beta \ddagger$ & $P$ valuet & Methylation $\Delta \beta \ddagger$ \\
\hline 21 & 42799141 & cg21549285 & $M X 1$ & Yes & $3.5 \mathrm{E}-139$ & -0.42 & $6.4 \mathrm{E}-83$ & -0.47 \\
\hline 3 & 122281881 & cg22930808 & PARP9 & Yes & $1.4 \mathrm{E}-105$ & -0.27 & $1.2 \mathrm{E}-74$ & -0.33 \\
\hline 21 & 42797588 & $\operatorname{cg} 22862003$ & $M X 1$ & Yes & $2.5 \mathrm{E}-126$ & -0.27 & $1.8 \mathrm{E}-77$ & -0.30 \\
\hline 1 & 79088769 & cg05696877 & IFI44L & Yes & $1.9 \mathrm{E}-120$ & -0.26 & $4.1 \mathrm{E}-86$ & -0.32 \\
\hline 1 & 79085586 & cg03607951 & IFI44L & Yes & $3.0 \mathrm{E}-141$ & -0.25 & $4.8 \mathrm{E}-83$ & -0.27 \\
\hline 10 & 91153143 & cg05552874 & IFIT1 & Yes & $2.5 \mathrm{E}-128$ & -0.25 & $1.8 \mathrm{E}-73$ & -0.27 \\
\hline 3 & 146260954 & cg06981309 & PLSCR1 & Yes & $4.9 E-157$ & -0.24 & $7.6 \mathrm{E}-91$ & -0.25 \\
\hline 3 & 122281975 & cg00959259 & PARPg & Yes & $9.3 \mathrm{E}-105$ & -0.23 & $6.1 \mathrm{E}-71$ & -0.27 \\
\hline 11 & 315102 & $\operatorname{cg} 23570810$ & IFITM1 & Yes & $1.6 \mathrm{E}-5$ & -0.20 & $3.4 \mathrm{E}-60$ & -0.24 \\
\hline 21 & 42797847 & cg26312951 & $M X 1$ & Yes & $1.3 \mathrm{E}-82$ & -0.18 & $1.2 E-59$ & -0.22 \\
\hline 22 & 50971140 & $\operatorname{cg} 20098015$ & $O D F 3 B$ & Yes & 7.1E-96 & -0.15 & $1.1 \mathrm{E}-52$ & -0.16 \\
\hline 2 & 7004578 & cg01028142 & CMPK2 & Yes & $1.2 \mathrm{E}-64$ & -0.15 & $4.5 \mathrm{E}-47$ & -0.19 \\
\hline 1 & 79085713 & cg17980508 & IFI44L & Yes & $3.8 \mathrm{E}-179$ & -0.14 & $1.1 \mathrm{E}-79$ & -0.13 \\
\hline 8 & 66751182 & cg14864167 & PDE7A & Yes & $3.6 \mathrm{E}-41$ & -0.14 & $4.0 \mathrm{E}-37$ & -0.20 \\
\hline 8 & 144099482 & $\operatorname{cg} 17052170$ & LOC100133669,LY6E & Yes & $1.5 \mathrm{E}-58$ & -0.13 & $2.6 \mathrm{E}-35$ & -0.15 \\
\hline 11 & 319667 & cg09122035 & Intergenic & NA & $3.1 \mathrm{E}-72$ & -0.13 & $9.9 \mathrm{E}-41$ & -0.13 \\
\hline 11 & 319555 & $\operatorname{cg} 20045320$ & Intergenic & NA & $1.0 \mathrm{E}-63$ & -0.13 & $8.3 \mathrm{E}-38$ & -0.13 \\
\hline 11 & 614761 & cg08926253 & IRF7 & Yes & $6.8 \mathrm{E}-81$ & -0.13 & $1.5 \mathrm{E}-54$ & -0.14 \\
\hline 1 & 79085162 & $\operatorname{cg} 13304609$ & IFI44L & Yes & $5.0 \mathrm{E}-63$ & -0.13 & $3.3 \mathrm{E}-47$ & -0.16 \\
\hline 6 & 35654363 & cg03546163 & FKBP5 & Yes & $1.5 \mathrm{E}-66$ & -0.13 & $1.8 \mathrm{E}-24$ & -0.11 \\
\hline 22 & 50973101 & cg05523603 & Intergenic & NA & 4.7E-71 & -0.13 & $2.0 \mathrm{E}-50$ & -0.14 \\
\hline 16 & 87371097 & cg01787084 & FBXO31 & NA & $1.4 \mathrm{E}-126$ & -0.13 & $8.3 \mathrm{E}-61$ & -0.10 \\
\hline 11 & 315262 & cg03038262 & IFITM1 & Yes & $1.6 \mathrm{E}-50$ & -0.12 & $2.7 \mathrm{E}-48$ & -0.17 \\
\hline 1 & 79118191 & cg01079652 & IFI44 & Yes & $3.6 \mathrm{E}-43$ & -0.12 & $1.7 \mathrm{E}-25$ & -0.13 \\
\hline 7 & 2444534 & $\operatorname{cg} 10152449$ & CHST12 & Yes & $3.6 \mathrm{E}-102$ & -0.11 & $3.5 \mathrm{E}-49$ & -0.10 \\
\hline 6 & 29911550 & $\operatorname{cg} 17608381$ & $H L A-A$ & Yes & $4.8 \mathrm{E}-25$ & -0.11 & $4.1 \mathrm{E}-15$ & -0.12 \\
\hline 2 & 7018020 & cg10959651 & RSAD2 & Yes & $2.9 \mathrm{E}-110$ & -0.11 & $3.1 \mathrm{E}-66$ & -0.12 \\
\hline 11 & 319718 & cg17990365 & IFITM3 & Yes & $9.5 \mathrm{E}-56$ & -0.11 & $1.5 \mathrm{E}-35$ & -0.11 \\
\hline 1 & 79085765 & cg00855901 & IFI44L & Yes & $1.0 \mathrm{E}-135$ & -0.11 & $2.6 \mathrm{E}-70$ & -0.11 \\
\hline 2 & 7016509 & $\operatorname{cg} 23213327$ & RSAD2 & Yes & 4.7E-92 & -0.10 & $8.0 \mathrm{E}-58$ & -0.10 \\
\hline 20 & 62204908 & cg01190666 & PRIC285 (HELZ2) & Yes & $1.2 \mathrm{E}-111$ & -0.10 & $5.7 \mathrm{E}-61$ & -0.11 \\
\hline 3 & 45984838 & cg08450017 & CXCR6,FYCO1 (RUFY3) & Yes & $1.4 \mathrm{E}-130$ & 0.13 & $1.8 \mathrm{E}-51$ & 0.10 \\
\hline
\end{tabular}

DMCs with $|\Delta \beta|>0.1$ in both the discovery and replication cohorts are listed.

${ }^{*}$ Database of interferon-regulated genes http://interferome.org.

tP value for case-control comparison using a linear regression model containing cell count estimates, age and sex as covariates.

$\ddagger$ Difference in average methylation beta value between patients and control individuals.

in the online Supplementary file 15). To increase the power to detect meQTLs for low frequency variants, the patients with SLE in the discovery and replication cohorts were combined for this analysis, as were the controls.

At $466 \mathrm{CpG}$ sites of the 7245 replicated DMCs in SLE, we observed evidence of genetic control in the form of meQTLs in patients with SLE or controls $\left(\mathrm{P}<6.5 \times 10^{-9}\right)$ (see table S10 in the online Supplementary file 16). To investigate whether the meQTL SNPs could inform genetic associations from studies on SLE, we compared their P values for association with SLE to the P values for all SNPs on the ImmunoChip in a case-control genetic association analysis in a larger set of Swedish patients with SLE and controls $\left(\mathrm{n}_{\mathrm{SLE}}=1135 ; \mathrm{n}_{\mathrm{ctrl}}=2931\right)$. We found that SNPs which are meQTLs for SLE-associated methylation changes were enriched for low $\mathrm{P}$ values in the genetic association analysis for SLE in our Swedish cohorts (figure 3). Among the SLE-associated meQTLs, we note seven GWAS risk loci for $\mathrm{SLE}^{34-36}$ : PTPRC (CD45), MHC-class III, UHRF1BP1, IRF5, IRF7, IKZF3 and UBE2L3 (see table S11 in the online Supplementary file 16). This suggests that variants at SLE risk loci may in part exert their influence on the phenotype through alteration of DNA methylation levels at regulatory regions of target genes. For example, at the UBE2L3 locus, the tested GWAS SNP is located downstream of the gene, but acts as a meQTL for an SLE associated DMC in the promoter of UBE2L3 (figure 4). For some of the SLE GWAS loci, the meQTL effect was observed in both patients and controls and in others exclusively in the patient or control group (see figure S6 in the online Supplementary file 18).

Lastly, we investigated whether SNPs affected the methylation variance at DMCs in SLE. We found that a small fraction of the 7245 DMCs in SLE had SNPs associated with variation in DNA methylation levels (var-meQTLs; 20 unique CpG sites, see table S12 in the online Supplementary file 19). The most significant var-meQTLs in both patients and control individuals were observed for one CpG site (cg07180897) in the major histocompatibility complex (MHC) class II gene $H L A-D Q B 2$, which is a known SLE risk locus. Nineteen of the 20 var-meQTL CpG sites also had meQTLs, that is, the genotype affected both the mean DNA methylation and variance of DNA methylation at these sites. 


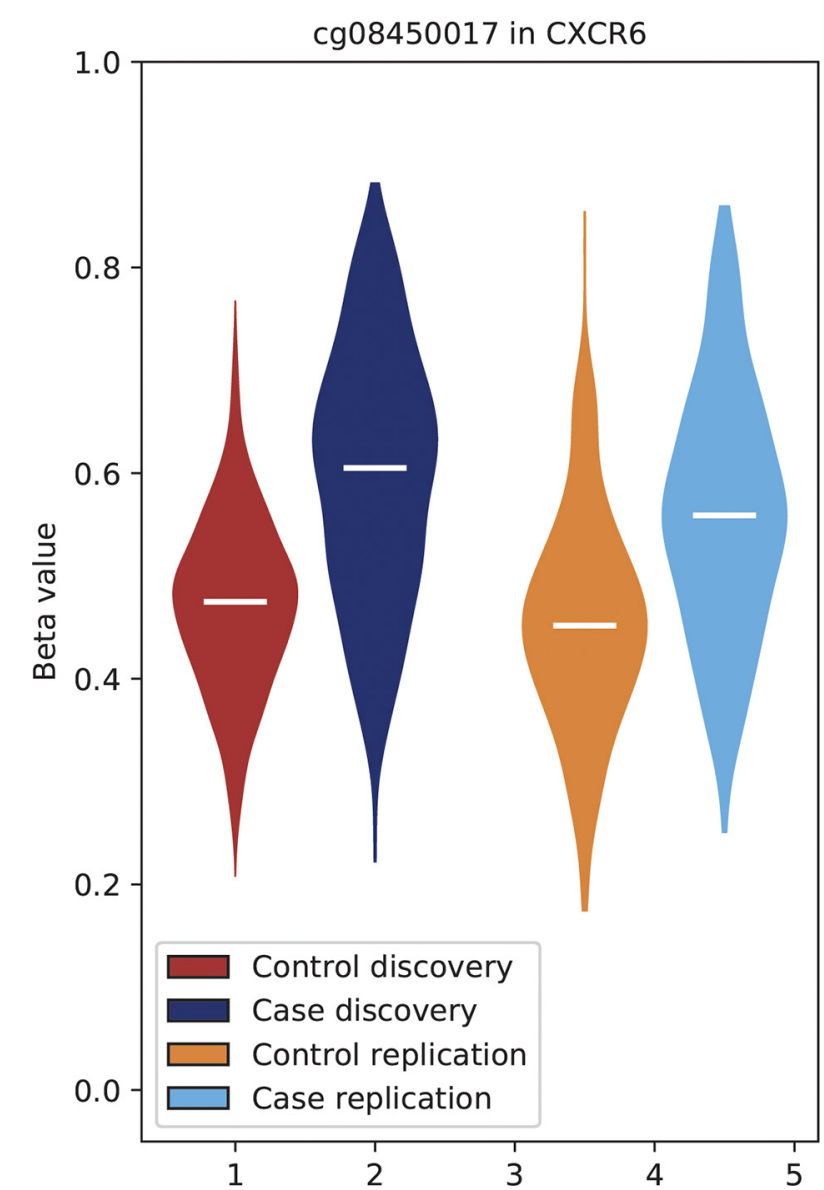

Figure 2 Violin plot of the DNA methylation levels at the CXCR6 gene in patients with systemic lupus erythematosus (SLE) and control individuals. Methylation levels at the CpG site cg08450017 in CXCR6 were increased in patients with SLE compared with controls in both the discovery and replication cohorts $\left(P\right.$ discovery $=1.4 \times 10^{-130}$ and $\mathrm{P}$ replication $\left.=1.8 \times 10^{-51}\right)$. Median methylation beta values are represented by the white horizontal lines in the violin plots.

\section{DISCUSSION}

We find wide-spread DNA methylation changes in SLE, the majority of which exhibit decreased methylation levels in patients compared with healthy controls. The top signals replicate previously reported associations in fractionated blood cells from patients with SLE, and we identify a large number of novel associations. Previous SLE methylation studies have been performed in smaller numbers of samples, which most likely is the reason for the large number of novel signals that we observe. Among $\mathrm{CpG}$ sites that have to our knowledge not previously been reported as epigenetically associated to SLE, we note multiple DMCs with increased methylation levels in SLE located in the promoter region of PDCD1 which encodes the PD-1 protein. PDCD1 acts an immune checkpoint receptor with a primary role in regulating $\mathrm{T}$ cell responses in order to maintain immune tolerance. Functional enrichment analyses indicate that the set of most significant DMCs in SLE are located in genes which play a role in regulating transcription in immune cells.

We observe a striking pattern of hypomethylation at IFN-signature genes, despite the fact that the majority of patients were inactive or under treatment at time of blood sampling. However, this IFN-pattern was more pronounced in patients with active disease. We have previously reported decreased methylation at IFN-induced genes also for primary Sjögren's syndrome. ${ }^{37} \mathrm{We}$ note that the pattern of hypomethylation at IFN-signature genes in blood is more pronounced in SLE, with patients with Sjögren's syndrome exhibiting average methylation levels which are intermediary to those of healthy individuals and patients with SLE. This is in line with gene expression studies showing an increased expression of IFN-induced genes in the vast majority of patients with SLE, ${ }^{38}$ while the IFN-signature is less prevalent in primary Sjögren's syndrome. ${ }^{39}$

The study was conducted on whole blood samples and we corrected our analysis for major blood cell types. To analyse the systemic components of autoimmunity, blood is thought to be the most appropriate sample type, while mechanisms of local inflammation at specific target organs would require analysis of additional tissue types. ${ }^{40}$ DNA extracted from whole blood is more readily available for analysis, but to fully decipher the contribution of DNA methylation variation in SLE, additional analyses of fractionated cells are needed. Such studies would have the ability to detect DNA methylation changes in SLE that are restricted to smaller cell subsets.

Despite previous reports of DMCs for ACR criteria, we were unable to formally replicate any of the associations with ACR criteria we observed in the discovery cohort. Reasons for the difference between this and previous studies could be the different cell types and different study designs that were used in the analyses. ${ }^{1416}$ Factors that complicate the analysis of altered DNA methylation in relation to the clinical criteria are that the SLE ACR criteria are collected cumulatively over a patient's disease course and that individual patients fulfil multiple criteria.

Table 2 Enrichment analyses of gene ontology (GO) terms based on the top 500 replicated differentially methylated CpG sites (according to association $\mathrm{P}$ value in the discovery cohort) with gene name annotation and the most significant $\mathrm{GO}$ terms are shown*

\begin{tabular}{|c|c|c|}
\hline & Molecules & $P$ value \\
\hline \multicolumn{3}{|l|}{ Molecular function } \\
\hline $\begin{array}{l}\text { Enzyme binding } \\
\text { GO:0019899 }\end{array}$ & 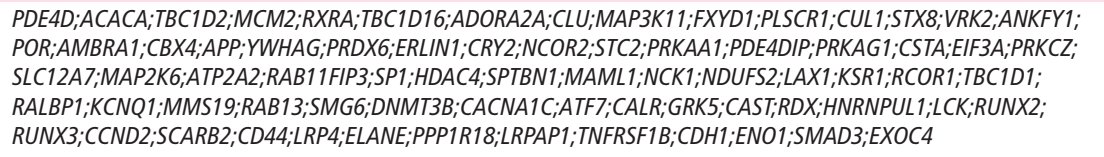 & $4.6 \mathrm{E}-06$ \\
\hline $\begin{array}{l}\text { Regulatory region } \\
\text { DNA binding } \\
\text { GO:0000975 }\end{array}$ & $\begin{array}{l}\text { BACH2;ZMYND8;ETS2;ACTN4;BCL11A;RREB1;RFX8;RXRA;GATAD2B;PRDX5;MNT;CBX4;CRY2;NCOR2;ZNF335;ZNF516; } \\
\text { CUX1;GABPB1;SP1;ZBTB16;ZNF148;HDAC4;IRF5;RCOR1;NR112;NFE2;NFIL3;EHF;ATF7;TCF12;ARID3A;NRF1;AKNA; } \\
\text { NR1H3;E2F3;RUNX2;RUNX1;RUNX3;IKZF4;LMO2;SMAD3 }\end{array}$ & 4.7E-06 \\
\hline \multicolumn{3}{|l|}{ Biological process } \\
\hline $\begin{array}{l}\text { Leucocyte activation } \\
\text { G0:0045321 }\end{array}$ & $\begin{array}{l}\text { PDCD1;HLA DMB;TUSC2;HLX;BCL11A;CD83;ADORA2A;PRAM1;FCER1G;CLU;FCGR3B;MAD1L1;FES;AIF1;PLSCR1; } \\
\text { PILRB;SSFTPD;ZBTB32;:PIK3R6;PRF1;ZNF335;PRKCZ;ZBTB16;IMPDH1;HDAC4;TNFSF13;AZU1;NCK1;LAX1;PTPRE; } \\
\text { ZFP36L1;DOCK2;LST1;RIPK3;NR1H3;LCK;RUNX2;LFNG;NLRP3;CORO1A;CD247;CD44;LY9;SMAD3;CDK6 }\end{array}$ & $3.8 \mathrm{E}-08$ \\
\hline
\end{tabular}




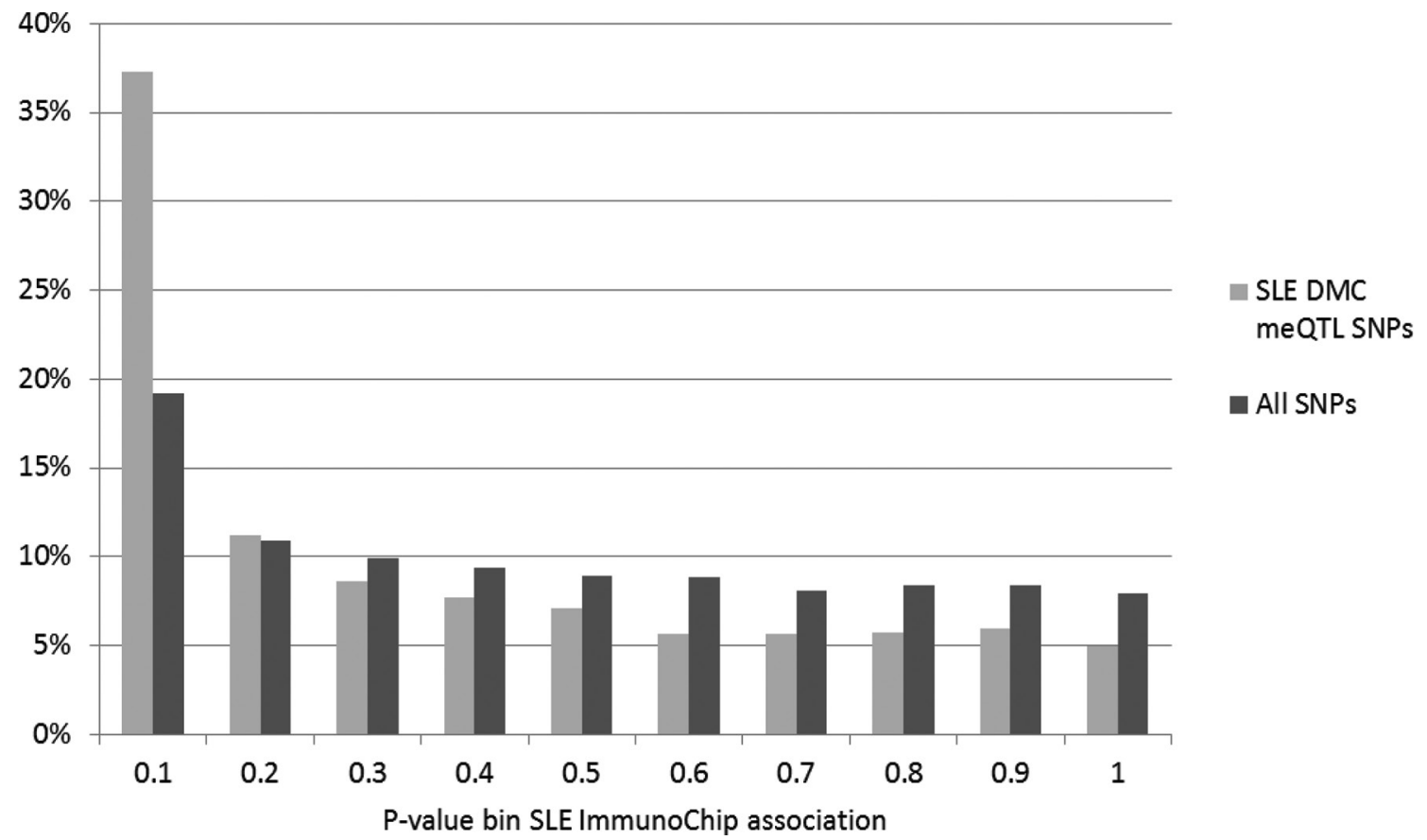

Figure 3 Enrichment of associated genetic variants in systemic lupus erythematosus (SLE) to methylation quantitative trait loci (meQTL) single nucleotide polymorphisms (SNPs) for differentially methylated CpG sites (DMCs) in SLE. The $x$-axis represents bins of P values from an SLE casecontrol genetic association analysis including 1135 Swedish patients with SLE and 2931 control individuals. Light grey bars represent SNPs which are significant meQTLs for CpG sites differentially methylated in SLE (466 CpG sites; 5307 SNPs). Bars in darker grey represent all SNPs on the ImmunoChip (133 838 quality controlled SNPs).

Longitudinal studies of DNA methylation would be useful in disentangling its role in clinical presentation of SLE.

Association with prescribed medications revealed a large number of affected $\mathrm{CpG}$ sites in patients treated with glucocorticoids. However, the majority of the observed DMCs in SLE were nominally significant also in the group of patients not treated with glucocorticoids. This indicates that the replicated SLE DMCs are not mainly driven by treatment effects. A previous study on the effects of systemic glucocorticoid exposure in patients with chronic obstructive pulmonary disease revealed that the majority of associated $\mathrm{CpG}$ sites had decreased methylation levels in treated patients, ${ }^{41}$ which is in line with the results presented here. Association of DNA methylation patterns with treatment may be confounded by the underlying cause for prescribing the drug and analyses of treatment effects on DNA methylation are hampered by high rates of medication non-adherence in SLE. ${ }^{42}$

It has previously been suggested for rheumatoid arthritis that DNA methylation could be a mediator of genetic risk in the disease, ${ }^{43}$ and we have recently reported genetic regulation of methylation at GWAS risk loci for Sjögren's syndrome. ${ }^{37}$ Similarly, we here observe evidence of genetic regulation of DNA

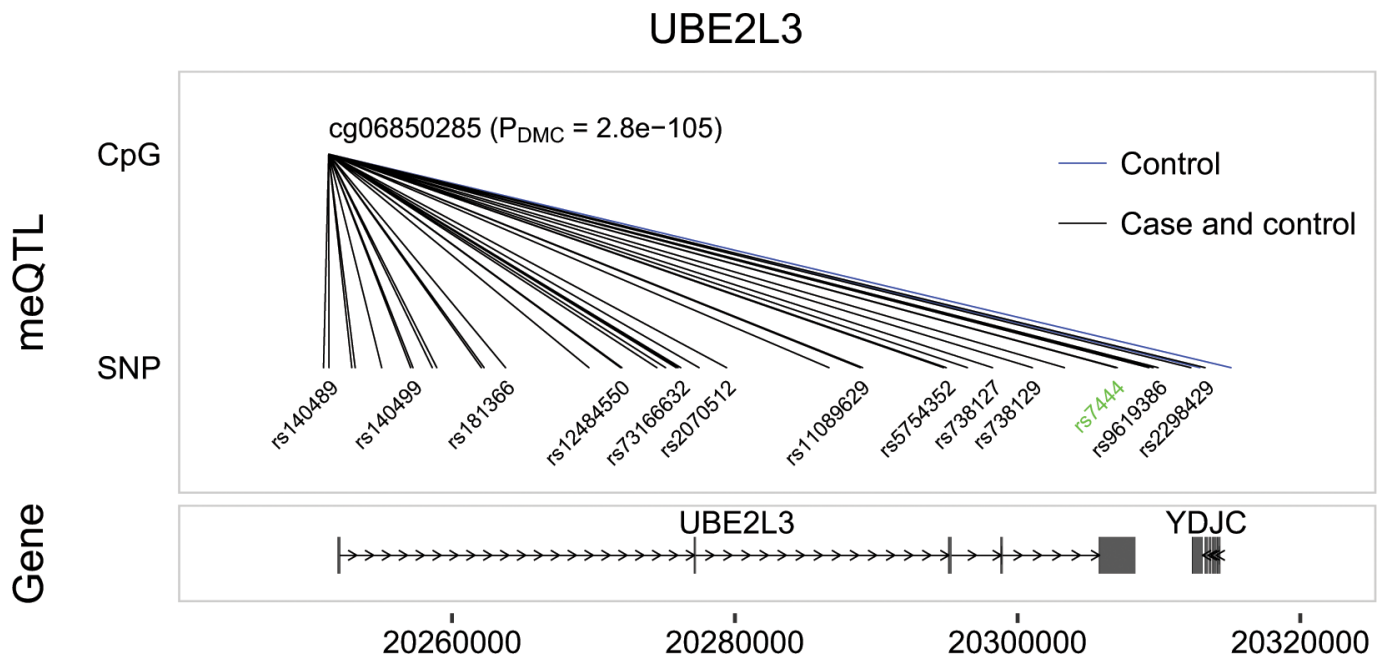

Figure 4 Illustration of genetic regulation of DNA methylation at the UBE2L3 genetic susceptibility locus for systemic lupus erythematosus (SLE) from a genome-wide association study (GWAS). The UBE2L3 locus on chromosome 22 with the differentially methylated CpG site (DMC) cg06850285 from the epigenome-wide association study is indicated at the top panel. The middle panel represents significant methylation quantitative trait loci (meQTLs) in controls only (illustrated by blue lines) or shared in both cases and controls (illustrated by black lines). The GWAS index single nucleotide polymorphism (SNP) is indicated in green. The bottom panel illustrates the RefSeq genes in the region. 
methylation at DMCs in SLE. Notably, we find GWAS variants associated with risk for SLE among the significant meQTLs, suggesting a functional mechanism for these genetic variants. However, since the coverage of CpG sites at SLE GWAS loci was low for the HM450k BeadChip, we have limited possibilities of fine-mapping the association signals. The fact that some meQTLs are observed exclusively in either the patient or control group suggests that a subset of the meQTLs that we detect are context dependent. These contexts could, for example, be differences in cell type composition as previously reported for eQTLs. ${ }^{44}$ The majority of meQTLs that we report are, however, shared between patients and controls. In contrast to genetic regulation of mean methylation levels which was observed for hundreds of $\mathrm{CpG}$ sites, we only observed genetic regulation of methylation variance at 20 DMCs in SLE. This suggests that genetic regulation of DNA methylation in SLE mainly is operating via effects on DNA methylation levels means, but that a smaller set of variants also have the ability to influence phenotype plasticity.

A main limitation of these data is that it is not possible to infer whether the methylation differences in SLE are causes or effects of the disease. Longitudinal studies will be required to completely elucidate the role of DNA methylation in SLE disease aetiology. In addition, it is possible that differences in proportions of cell subtypes affected the results. Another limitation is that only methylation at a defined fraction of all $\mathrm{CpG}$ sites in the genome was analysed. Alternative approaches such as whole genome bisulfite sequencing of fractionated cells have the potential to fully characterise the epigenetic landscape in SLE. Epigenetic variants could be the starting point for developing novel epigenetic biomarkers to improve diagnosis in SLE, and the reversible nature of epigenetic marks suggests them as potential targets for therapeutic interventions.

Acknowledgements We thank Rezvan Kiani, Marianne Petersson and Karolina Tandre for collecting samples from patients and controls and medicinska Biobanken Norr for providing samples from control individuals. Genotyping and DNA methylation analyses were performed at the SNP\&SEQ Technology Platform at the National Genomics Infrastructure (NGI) hosted by Science for Life Laboratory in Uppsala, Sweden (www.genotyping.se; www.sequencing.se). We thank Tomas Axelsson, Anna Haukkala and Anders Lundmark for excellent technical assistance. We especially thank all patients and blood donors who donated samples to this study.

Contributors JI-K, A-CS, JKS designed the study and drafted the manuscript. DL, GN, M-LE, SR-D, AAB, AJ, LP, IG, ES, CS and LR collected patient and control material and clinical data. JI-K and JKS performed the experiments. JI-K, JKS, AA and JCA analysed the data. All authors read and provided critical review and accepted the final version of the manuscript.

Funding This study was supported by grants from the Knut and Alice Wallenberg Foundation (KAW 2011.0073), the Swedish Research Council for Medicine and Health (VR-MH Dnr 521-2014-2263 to ACS, Dnr 521-2013-2830 to LR, Dnr 5212014-3954 to ES and Dnr 2016-01982 to GN), an AstraZeneca-Science for Life Laboratory research collaboration grant (to LR), the Swedish Society for Medical Research (to CS), the Swedish Rheumatism Association (to CS and DL), King Gustaf V's 80th Birthday Fund (to DL) and a Swedish Research Council postdoc grant (VR Dnr 350-2012-256 to JKS). Funding to collect samples and characterise patients/ controls from the Karolinska University hospital was provided by the Swedish HeartLung foundation (ES), Stockholm County Council (ALF) (ES and IG), King Gustaf V's 80th Birthday Fund (ES and IG) and the Swedish Rheumatism Association (ES and IG). The SNP\&SEQ Technology Platform is supported by Science for Life Laboratory, the Knut and Alice Wallenberg Foundation and the Swedish Research Council (VR$\mathrm{RFI).}$

\section{Competing interests None declared.}

Patient consent Obtained.

Ethics approval The study was approved by the Regional Ethics board in Uppsala with Dnr 00-227 and 2016/155.

Provenance and peer review Not commissioned; externally peer reviewed.

Data sharing statement Normalised or raw intensity data of the HM450k BeadChips are available upon request from the authors on a collaborative basis.
Open Access This is an Open Access article distributed in accordance with the Creative Commons Attribution Non Commercial (CC BY-NC 4.0) license, which permits others to distribute, remix, adapt, build upon this work non-commercially, and license their derivative works on different terms, provided the original work is properly cited and the use is non-commercial. See: http://creativecommons.org/ licenses/by-nc/4.0/

(c) Article author(s) (or their employer(s) unless otherwise stated in the text of the article) 2018. All rights reserved. No commercial use is permitted unless otherwise expressly granted.

\section{REFERENCES}

1 Bengtsson AA, Rönnblom L. Systemic lupus erythematosus: still a challenge for physicians. J Intern Med 2017;281:52-64.

2 Hagberg N, Rönnblom L. Systemic lupus erythematosus - a disease with a dysregulated type I interferon system. Scand J Immunol 2015;82:199-207.

3 Chen L, Morris DL, Vyse TJ. Genetic advances in systemic lupus erythematosus: an update. Curr Opin Rheumatol 2017;29:423-33.

4 Langefeld CD, Ainsworth HC, Cunninghame Graham DS, et al. Transancestral mapping and genetic load in systemic lupus erythematosus. Nat Commun 2017;8:16021.

5 Deng Y, Tsao BP. Advances in lupus genetics and epigenetics. Curr Opin Rheumatol 2014; 26:482-92.

6 Teruel M, Alarcón-Riquelme ME. Genetics of systemic lupus erythematosus and Sjögren's syndrome: an update. Curr Opin Rheumatol 2016;28:506-14.

7 Lu Q, Qiu X, Hu N, et al. Epigenetics, disease, and therapeutic interventions. Ageing Res Rev 2006:5:449-67.

8 Javierre BM, Fernandez AF, Richter J, et al. Changes in the pattern of DNA methylation associate with twin discordance in systemic lupus erythematosus. Genome Res 2010;20:170-9.

9 Richardson B, Scheinbart L, Strahler J, et al. Evidence for impaired T cell DNA methylation in systemic lupus erythematosus and rheumatoid arthritis. Arthritis Rheum 1990;33:1665-73.

10 Rubin RL. Drug-induced lupus. Expert Opin Drug Saf 2015;14:361-78.

11 Absher DM, Li X, Waite LL, et al. Genome-wide DNA methylation analysis of systemic lupus erythematosus reveals persistent hypomethylation of interferon genes and compositional changes to CD4+ T-cell populations. PLoS Genet 2013;9:e1003678

12 Coit P, Jeffries M, Altorok N, et al. Genome-wide DNA methylation study suggests epigenetic accessibility and transcriptional poising of interferon-regulated genes in naïve CD4+ T cells from lupus patients. J Autoimmun 2013;43:78-84.

13 Chung SA, Nititham J, Elboudwarej E, et al. Genome-Wide Assessment of Differential DNA Methylation Associated with Autoantibody Production in Systemic Lupus Erythematosus. PLoS One 2015;10:e0129813.

14 Coit $P$, Renauer $P$, Jeffries MA, et al. Renal involvement in lupus is characterized by unique DNA methylation changes in naïve CD4 $+\mathrm{T}$ cells. J Autoimmun 2015;61:29-35.

15 Renauer $\mathrm{P}$, Coit $\mathrm{P}$, Jeffries MA, et al. DNA methylation patterns in naïve CD4+ T cells identify epigenetic susceptibility loci for malar rash and discoid rash in systemic lupus erythematosus. Lupus Sci Med 2015;2:e000101.

16 Mok A, Solomon O, Nayak RR, et al. Genome-wide profiling identifies associations between lupus nephritis and differential methylation of genes regulating tissue hypoxia and type 1 interferon responses. Lupus Sci Med 2016:3:e000183.

17 Ighe A, Dahlström Ö, Skogh T, et al. Application of the 2012 Systemic Lupus International Collaborating Clinics classification criteria to patients in a regiona Swedish systemic lupus erythematosus register. Arthritis Res Ther 2015;17:3.

18 Tan EM, Cohen AS, Fries JF, et al. The 1982 revised criteria for the classification of systemic lupus erythematosus. Arthritis Rheum 1982;25:1271-7.

19 Bibikova M, Barnes B, Tsan C, et al. High density DNA methylation array with single CpG site resolution. Genomics 2011;98:288-95.

20 R Core Team. R: a language and environment for statistical computing: R Foundation for Statistical Computing. Vienna, Austria: R Core Team, 2016.

21 Aryee MJ, Jaffe AE, Corrada-Bravo H, et al. Minfi: a flexible and comprehensive Bioconductor package for the analysis of Infinium DNA methylation microarrays. Bioinformatics 2014;30:1363-9.

22 Maksimovic J, Gordon L, Oshlack A. SWAN: Subset-quantile within array normalization for illumina infinium HumanMethylation450 BeadChips. Genome Biol 2012;13:R44.

23 Cortes A, Brown MA. Promise and pitfalls of the Immunochip. Arthritis Res Ther 2011;13:101.

24 Houseman EA, Accomando WP, Koestler DC, et al. DNA methylation arrays as surrogate measures of cell mixture distribution. BMC Bioinformatics 2012;13:86.

25 Purcell S, Neale B, Todd-Brown K, et al. PLINK: a tool set for whole-genome association and population-based linkage analyses. Am J Hum Genet 2007:81:559-75

26 Qin M, Guo Y, Jiang L, et al. Elevated levels of serum SCXCL16 in systemic lupus erythematosus; potential involvement in cutaneous and renal manifestations. Clin Rheumatol 2014;33:1595-601.

27 Wen S, He F, Zhu X, et al. IFN- $\gamma$, CXCL16, uPAR: potential biomarkers for systemic lupus erythematosus. Clin Exp Rheumatol 2017 [Epub ahead of print 16 Jun 2017]. 
28 Coit P, Yalavarthi S, Ognenovski M, et al. Epigenome profiling reveals significant DNA demethylation of interferon signature genes in lupus neutrophils. J Autoimmun 2015;58:59-66.

29 Zhu H, Mi W, Luo H, et al. Whole-genome transcription and DNA methylation analysis of peripheral blood mononuclear cells identified aberrant gene regulation pathways in systemic lupus erythematosus. Arthritis Res Ther 2016;18:162.

30 Prokunina L, Castillejo-López C, Oberg F, et al. A regulatory polymorphism in PDCD1 is associated with susceptibility to systemic lupus erythematosus in humans. Nat Genet 2002;32:666-9.

31 Gao J, Gai N, Wang L, et al. Meta-analysis of programmed cell death 1 polymorphisms with systemic lupus erythematosus risk. Oncotarget 2017;8:36885-97.

32 Okazaki T, Wang J. PD-1/PD-L pathway and autoimmunity. Autoimmunity 2005;38:353-7.

33 Bierie B, Edwin M, Melenhorst JJ, et al. The proliferation associated nuclear element (PANE1) is conserved between mammals and fish and preferentially expressed in activated lymphoid cells. Gene Expr Patterns 2004;4:389-95.

34 Morris DL, Sheng Y, Zhang Y, et al. Genome-wide association meta-analysis in Chinese and European individuals identifies ten new loci associated with systemic lupus erythematosus. Nat Genet 2016;48:940-6.

35 Bentham J, Morris DL, Graham DSC, et al. Genetic association analyses implicate aberrant regulation of innate and adaptive immunity genes in the pathogenesis of systemic lupus erythematosus. Nat Genet 2015;47:1457-64.

36 Welter D, MacArthur J, Morales J, et al. The NHGRI GWAS Catalog, a curated resource of SNP-trait associations. Nucleic Acids Res 2014;42:D1001-6.
37 Imgenberg-Kreuz J, Sandling JK, Almlöf JC, et al. Genome-wide DNA methylation analysis in multiple tissues in primary Sjögren's syndrome reveals regulatory effects at interferon-induced genes. Ann Rheum Dis 2016;75:2029-36.

38 Chiche L, Jourde-Chiche N, Whalen E, et al. Modular transcriptional repertoire analyses of adults with systemic lupus erythematosus reveal distinct type I and type II interferon signatures. Arthritis Rheumatol 2014;66:1583-95.

39 Brkic Z, Maria NI, van Helden-Meeuwsen CG, et al. Prevalence of interferon type I signature in CD14 monocytes of patients with Sjogren's syndrome and association with disease activity and BAFF gene expression. Ann Rheum Dis 2013;72:728-35.

40 Cole MB, Quach H, Quach D, et al. Epigenetic signatures of salivary gland inflammation in sjögren's syndrome. Arthritis Rheumatol 2016:68:2936-44.

41 Wan ES, Qiu W, Baccarelli A, et al. Systemic steroid exposure is associated with differential methylation in chronic obstructive pulmonary disease. Am J Respir Crit Care Med 2012;186:1248-55.

42 Costedoat-Chalumeau N, Pouchot J, Guettrot-Imbert G, et al. Adherence to treatment in systemic lupus erythematosus patients. Best Pract Res Clin Rheumatol 2013;27:329-40.

43 Liu Y, Aryee MJ, Padyukov L, et al. Epigenome-wide association data implicate DNA methylation as an intermediary of genetic risk in rheumatoid arthritis. Nat Biotechnol 2013;31:142-7.

44 Zhernakova DV, Deelen P, Vermaat M, et al. Identification of context-dependent expression quantitative trait loci in whole blood. Nat Genet 2017;49:139-45 